\title{
Correlation of Tumour Response with Starting Tumour Size and Dose of Tamoxifen in an N-Methyl-N-Nitrosourea (NMU)- Induced Rat Mammary Cancer Model
}

\author{
Hassan Muhammad Yankuzo ${ }^{1}$, Sharifah Tuan Sheriff Emilia², Rumaizi Shaari ${ }^{3}$, \\ Nik Soriani Yaacob ${ }^{1 *}$
}

\begin{abstract}
Background: The aim of this preliminary study was to address variations of responses observed with different starting tumor sizes of 10 and $15 \mathrm{~mm}$, and the effects of different doses of tamoxifen (TAM) on experimental rat mammary tumors. Materials and Methods: Thirty-five inbred female Sprague Dawley rats aged 43 days were administered with three weekly doses of $\mathrm{N}$-methyl-N-nitrosourea (NMU) intraperitoneally (ip) at $50 \mathrm{mg} / \mathrm{kg}$ body weight. Animals were randomized (beginning from $10 \mathrm{~mm}$ tumor size) into four TAM-treated $(50,100,200$ and $500 \mu \mathrm{g} / \mathrm{day})$ groups of six animals each, and another group $(\mathrm{n}=6)$ treated with TAM $100 \mu \mathrm{g} / \mathrm{day}$ at starting tumour size of $15 \mathrm{~mm}$. The animals were treated by oral gavage daily for 8 weeks before sacrifice. Results: Serum urea and creatinine, and overall physical tumor burden were significantly modulated in animals treated with variable doses of TAM compared to the untreated controls $(n=5)$. Final body weight and tumor number were significantly different in the $10 \mathrm{~mm}$-treated animals compared to those treated at $15 \mathrm{~mm}$. There were no significant differences in histopathological features among all the groups. Conclusions: Our findings suggest the importance of standardizing tumour size and drug doses before initiation of treatment, particularly in the direct comparison of basic end-tumour physical parameters.
\end{abstract}

Keywords: NMU - rat mammary tumor - tamoxifen - tumor size

Asian Pac J Cancer Prev, 15 (16), 6721-6726

\section{Introduction}

Breast cancer is the commonest cancer among women in the United States and the second leading cause of cancer deaths in the women worldwide (after lung cancer) with estimated 232, 340 new cases of invasive breast cancer and 39,620 deaths among US women in 2013 (De Santis et al., 2013). However, the global trend of breast cancer burden varies between regions and countries. According to the report of Porter (Porter, 2009), the trend of breast cancer burden was particularly more evident in the lowand middle-income countries where $45 \%$ incidence rate and $55 \%$ breast cancer- related deaths were estimated from over 1 million cases diagnosed worldwide in 2009.

In Malaysia, breast cancer is also the commonest cancer constituting $18.1 \%$ of the reported cancer cases, and the leading cause of cancer related mortality among women within 40-49 years (Yip et al., 2006). It has been estimated that 1 out of every 20 women in Malaysia had 1 in 20 chance of developing breast cancer in her lifetime, and the incidence was highest in the Chinese, followed by Indians and Malays (Rampal and Yahaya, 2008; Dahlui et al., 2011; Moore, 2013).

Experimental animal models (particularly rodents) are commonly used in the study of breast cancer because of their biological similarity with human breast cancer in terms of epithelial origin, hormonal (estrogen) dependence and gene expression profiles (Chan et al., 2005). Chemically-induced breast cancer rat model is notably a simple and standard laboratory model for the study of human breast cancer (Tsubura et al., 2011). For such model, the chemical carcinogens N-Methyl-NNitrosourea (NMU) and 7, 12-dimethyl-benzanthracene (DMBA), are most frequently used for breast cancer induction. NMU is particularly known to induce estrogendependent mammary tumours in female Sprague Dawley rats (Soares-Maia et al., 2013).

Tamoxifen (TAM) has been the gold standard antiestrogen drug of choice for prevention and treatment of hormone dependent breast cancer in the high risk women (Jordan, 2012). But TAM is also known to have many side effects (Karimi et al., 2009) that limit its routine clinical application. Studies on the effects of TAM on experimental rat mammary tumours have been reported using variable

${ }^{1}$ Departments of Chemical Pathology and ${ }^{2}$ Pathology, School of Medical Sciences, Universiti Sains Malaysia, Kubang Kerian, ${ }^{3}$ Department of Surgery, Faculty of Veterinary Medicine, Universiti Malaysia Kelantan, Kelantan, Malaysia *For correspondence: niksoriani@usm.my 
doses of the antiestrogen. Most of these studies have focused on the chemoprevention effects (Manni et al., 2010) or complications of long term adjuvant treatment, including development of TAM resistance (Motamedi et al., 2012) and tendencies to induce carcinogenic effects (Behtash et al., 2009).

Tumour size is an established independent predictor in the management of breast cancer patients (Orang et al., 2013; Chin et al., 2014). However unlike in the clinical setting, it is interesting that convenient tumour size for initiating treatment on experimental animal models can easily be standardized. Yet variable methodologies, particularly the starting tumour size and dose of TAM have been reported in many literature reports; which lead to some degree of inconsistencies on the end-tumour response data. It was reported that treatments were mostly initiated at starting tumour sizes between $5 \mathrm{~mm}$ to $15 \mathrm{~mm}$ (Goss et al., 2007; Nishino et al., 2009; Ahlem et al., 2011).

To our knowledge to date, no experimental rat mmmary tumor-regression study has been conducted specifically to correlate the effect of variations in starting tumor size with the end-tumor response data. Thus this study was initiated to help clarify the variations of TAM dose and tumor size in NMU-induced rat mammary tumor model at the start of treatment. The study aimed to highlight the importance of standardizing starting tumor size and drug dose before initiation of treatment with potential anticancer agents. This may help to achieve uniform end-tumor response data and also improve the natural course of treatment response.

\section{Materials and Methods}

\section{Animals}

Inbred female Sprague Dawley rats (Rattus norvegicus) (body weight $100 \mathrm{~g}$ to $150 \mathrm{~g}$ ) were obtained from the Animal Research and Service Centre (ARASC), Universiti Sains Malaysia (USM). The animals were housed (in pairs) in the polycarbonate cages with 2 inches thick of pinewood chip bedding for absorbtion of their excreta. The rats were acclimatized to standard vivarium conditions (temperature $23 \pm 2^{\circ} \mathrm{C}$, relative humidity $70 \pm 5 \%$, and 14 h light-10 h dark cycle) for 1 week. They were fed with rodent pellet (Gold Coin Feedmills Sdn. Bhd. Malaysia) and had free access to tap water ad libitum. Environmental enrichment using tissue paper rolls and rubber toys were occasionally provided to the animals. The study was performed following the standards and guidelines as approved by the USM Animal Ethics Committee with reference number USM/AEC/2011/(69)(304).

\section{Induction of breast cancer}

Breast cancer was induced by NMU (Sigma, MO, USA), injected intraperitoneally in three weekly doses of $50 \mathrm{mg} / \mathrm{kg}$ body weight starting from 43 days of age. NMU was freshly dissolved in a warm physiologic saline (25 mg/ml), adjusted to $\mathrm{pH} 4.0$ using $3 \%$ acetic acid and maintaned at $35-40^{\circ} \mathrm{C}$ just before administration. The agematched control rats received $0.9 \%$ physiologic saline. All rats were weighed weekly following carcinogen treatment, and their mammary glands were palpated twice weekly for detection of tumours starting from 3 weeks after the first
NMU injection. Palpable tumours appeared within 6-9 weeks post induction and tumour growth was measured using a digital vernier caliper. Tumour number, size and volume were also recorded weekly.

\section{Experimental design}

Two experimental protocols were utilized. The first protocol was to compare the effect of variable doses $(50,100,200$, and $500 \mu \mathrm{g})$ of TAM. For this purpose animals were randomised (at $10 \mathrm{~mm}$ tumour size) into four treatment groups ( $n=6$ per group) and an untreated control group $(n=5)$. Tamoxifen (free base, Sigma, MO, USA) solution was prepared by first dissolving in absolute ethanol $(10 \mathrm{mg} / \mathrm{ml})$ followed by suspension in the corn oil. The ethanol was evaporated under low stream nitrogen gas before use. Daily doses were aliquoted in small eppendorf tubes covered with aluminum foil and kept at $-20^{\circ} \mathrm{C}$. TAM was administered by oral gavage daily for eight weeks, beginning at the initial tumour size of $10 \mathrm{~mm}$. Response to treatment was assessed weekly by measurement of the animal body weight and tumour growth variables.

Next protocol was to compare the end tumour responses in animals that were initiated treatment with TAM $100 \mu \mathrm{g} /$ day for 8 weeks at the starting tumour size of $10 \mathrm{~mm}$ and $15 \mathrm{~mm}$. To achieve this, a group of animals $(n=6)$ were treated with TAM $100 \mu \mathrm{g}$ daily for 8 weeks at the starting tumor size of $15 \mathrm{~mm}$ and compared with the ones that begin treatment at $10 \mathrm{~mm}$ tumour size.

Existing tumours at the start of treatment were closely monitored throughout the study period, and the appearance and growth of new tumours were recorded and followed up. Untreated animals were sacrificed 8 weeks after detection of a $10 \mathrm{~mm}$ tumour or earlier if the tumour size reached $40 \mathrm{~mm}$. All rats were monitored for signs of physical distress (e.g., reduced food and water intake, labored breathing, restricted movements and persistent recumbence posture). Other major complications like infected ulcer on the tumour surface, high tumour burden impairing normal movement of the animal, bleeding orifices and/or significant weight loss (more than $20 \%$ weight lost after induction) were also monitored. In the event of any complications, appropriate interventions under supervision of veterinary specialists were offered where necessary.

\section{Tumor volume measurement}

To analyze changes in tumour growth following treatment with TAM, the tumour volume was monitored weekly by measuring tumour length (a) and width (b) (in millimeter) at right angles using a digital Vernier caliper. The tumour volume $\left(\mathrm{mm}^{3}\right)$ was estimated using Carlson's formula $V=a b^{2} / 2$, where " $a$ " and " $b$ " represent the longest and shortest tumour diameters respectively. The tumour volume for each rat was quantified as total volume of all sizeable nodules in order to represent the actual tumour burden.

\section{Animals sacrifice and samples preparation}

Animals were euthanized after 8 weeks of treatment using i.p. phenobarbitone (Alfasan, BV, Holland) at a dose of $50 \mathrm{mg} / \mathrm{kg}$. Blood was immediately collected by 
cardiac puncture into plane vacutainer tubes and allowed to coagulate for about 1 hour at room temperature. The blood was centrifuged at $10,000 \times \mathrm{g}$ for 10 minutes at $4^{\circ} \mathrm{C}$ to separate the serum, plasma, and red blood cells. Serum analyses of liver enzymes (Alanine and aspartate transaminases), renal function profile (electrolytes, urea, creatinine, uric acid), and calcium levels were conducted using automated chemistry analyzer (Architect, c8000) to ascertain side effects from our interventions, if any.

In order to define and compare histopathological changes in the mammary tumours after 8 weeks of treatment, representative sections of each group were subjected to routine haematoxylin and eosin (H \& E) staining. Briefly, the excised tumours were fixed in $10 \%$ neutral buffered formalin for 24 hours and processed overnight for paraffin embedding. Sections of $3 \mu \mathrm{m}$ were cut, rehydrated in xylene and graded ethanol solutions and stained with $\mathrm{H} \& \mathrm{E}$. The specimens were examined by a pathologist and changes were graded according to Nottingham modification of the Bloom and Richardson method, where extent of tubule formation, nuclear pleomorphism and abnormal mitotic figures were evaluated (Meyer et al., 2005).

\section{Statistical analysis}

Data analysis was conducted using SPSS Version 20 (IBM Corp) and the results for tumour growth variables and animal body weight were expressed as median and interquartile range (IQR). Multiple Man Whitney test with Bonferroni correction was used to compare differences in the end tumour burden between untreated tumour bearing animals (controls) and animals treated with variable doses of TAM. Statistical differences between starting treatment at $10 \mathrm{~mm}$ and $15 \mathrm{~mm}$ starting tumour size were compared by using Man Whitney test. Comparisons with $\mathrm{p}<0.05$ were considered to be statistically significant.

\section{Results}

Effect of variable doses of TAM on the end tumour burden Animals treated with 100,200 and 500 $\mu \mathrm{g}$ TAM show significant dose-dependent reduction in the final number of tumor nodules and the end tumor volume compared to the untreated controls (Table 1). The values of mean tumor weight at necropsy $(1.25 \pm 0.35,0.31 \pm 5.89,0.00 \pm 2.17)$ were also reduced (although not statistically significant) compared to the untreated group $(2.14 \pm 9.12)$. In contrast the animals treated with TAM $50 \mu \mathrm{g}$ /day did not show significant effect on any of the tumour growth parameters measured compared to untreated controls. Generally all animals had marginal increase in body weight throughout the experiment and comparison of mean change in body weights show non-uniform disparity between TAM-treated and untreated groups.

Nonetheless, two rats each from the group treated with TAM $50 \mu \mathrm{g} /$ day and $200 \mu \mathrm{g} /$ day, developed infected ulcer at about 7 weeks of treatment and subsequently lost significant body weight. These animals were thus humanely euthanized. The remaining NMU-induced rats without tumour formation after 25 weeks post induction were excluded from the analysis

\section{Effect of variable doses of TAM on biochemical parameters}

Serum renal profile, levels of liver enzymes and calcium were determined to assess any possible side effects resulting from the intervention. However, no significant changes were observed in all the parameters above between TAM-treated animals and the untreated controls (data not shown).

\section{Histopathological changes in NMU-induced rat mammary tumors}

The $\mathrm{H} \& \mathrm{E}$ staining of the representative mammary tumour sections (Figure 1) indicated invasive adenocarcinomas in both untreated control group and the tumor sections treated with variable doses of TAM. However, the phenotype in the treated groups were of slightly less aggressive subtypes (cribriform carcinoma) compared to that of untreated control. Cribriform carcinoma showed clusters of epithelial cells with intense desmoplasia and lymphocytic infiltrations at the periphery. In addition, most of the sections displayed numerous cystic changes typical of human adenoid cystic carcinoma (ACC), a rare type of breast cancer comprising of about

Table 1. Effect of Variable doses of TAM on the end Tumor Burden Median (IQR)

\begin{tabular}{lcccc}
\hline Group & Final tumour number & Change in tumour volume $\left(\mathrm{mm}^{3}\right)$ & Tumour weight $(\mathrm{g})$ & Change of body weight $(\mathrm{g})$ \\
\hline Untreated & $2.0(3.0)$ & $1,225.0(24,349.0)$ & $2.1(9.1)$ & $25.0(82.0)$ \\
TAM 50 & $2.0(2.0)$ & $770.0(3,189.0)$ & $2.1(9.4)$ & $-1.5(22.0)$ \\
TAM 100 & $1.0(1.0)^{*}$ & $-46.0(127.0)^{*}$ & $1.3(0.4)$ & $28.0(12.0)$ \\
TAM 200 & $0.0(1.0)^{*}$ & $-173.0(489.0)^{*}$ & $0.3(5.9)$ & $-1.0(21.0)$ \\
TAM 500 & $0.0(1.0)^{*}$ & $-149.0(123.0)^{*}$ & $0.0(2.2)$ & $3.0(21.0)$ \\
\hline
\end{tabular}

Data were analyzed by Multiple Man Whitney with Bonferroni correction ( $\alpha / 4$ comparisons); *Significantly different from untreated control (p<0.05); TAM 50: Tamoxifen $50 \mu \mathrm{g}$ /day; TAM 100: Tamoxifen $100 \mu \mathrm{g} /$ day; TAM 200: Tamoxifen $200 \mu \mathrm{g} /$ day; TAM 500: Tamoxifen $500 \mu \mathrm{g} /$ day; Negative score implies significant shrinkage of the tumour at the end of treatment compared to the initial tumour volume

Table 2. Comparison of end Tumor Response according to the Starting Tumor Size

\begin{tabular}{lcccc}
\hline Group & Final tumour number & Change in tumour volume $\left(\mathrm{mm}^{3}\right)$ & Tumour weight $(\mathrm{g})$ & Change of body weight $(\mathrm{g})$ \\
\hline Untreated & $2.0(3.0)$ & $1,225.0(24,349.0)$ & $2.1(9.12)$ & $25.0(82.0)$ \\
$10 \mathrm{~mm}$ & $1.0(1.0)^{*}$ & $-46.0(127.0)$ & $1.3(0.4)$ & $28.0(12.0)^{*}$ \\
$15 \mathrm{~mm}$ & $2.0(2.0)$ & $1,371.0(8,072.0)$ & $4.4(6.5)$ & $2.5(12.0)$ \\
\hline
\end{tabular}

Data were analyzed by Man-Whitney test; *Significantly different from $15 \mathrm{~mm}$-treated animals (p<0.05); $10 \mathrm{~mm}$ : Animals initiated on TAM $100 \mu \mathrm{g} /$ day at starting tumor size of $10 \mathrm{~mm} ; 15 \mathrm{~mm}$ : Animals initiated on TAM $100 \mu \mathrm{g} /$ day at starting tumor size of $15 \mathrm{~mm}$ 


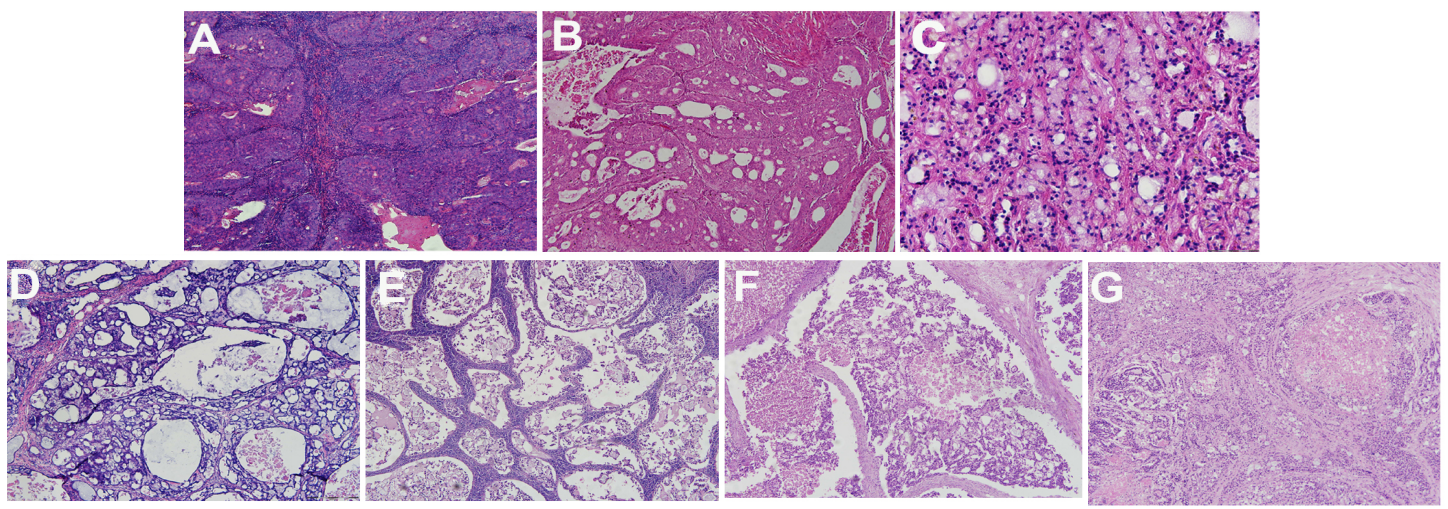

Figure 1. NMU-Induced Rat Mammary Tumor Sections Stained with Hematoxylin \& Eosin. No significant histomorphological variations between untreated tumors and animals (bearing NMU-induced mammary tumors) treated with variable doses of TAM at starting tumor size of 10 or $15 \mathrm{~mm}$. Mammary tumors were harvested after 8 weeks of treatment with variable doses of TAM, and representative sections from each group were stained with hematoxylin \& Eosin ( $\mathrm{H} \& \mathrm{E})$ for comparison purposes as described in the materials and methods section. (a-b) Sections of tumor untreated at $10 \mathrm{~mm}$ and $15 \mathrm{~mm}$ tumor sizes, $\mathrm{n}=5$ rats. (c) Tumor section of the rats treated with TAM $50 \mu \mathrm{g} /$ day showing grade 2 invasive carcinoma with prominent cystic changes. (d-e) Tumor sections of the rats treated with TAM $100 \mu \mathrm{g} /$ day at starting tumor sizes of $10 \mathrm{~mm}$ and $15 \mathrm{~mm}$, features were consistent with grade 2 invasive carcinoma, with additional moderate foci of central necrosis (comedo type of necrosis). (f-g) Sections of relapsed tumors from the rats treated with TAM 200 and $500 \mu \mathrm{g} /$ day showing many foci of central necrosis and other features consistent with grade 2 invasive carcinoma. All sections were captured at $10 \mathrm{x}$ magnification, and scored by modified Scarrf-Bloom-Richardson (SBR) scoring index. (c-g) $n=6$ rats per group

$0.1 \%$ human breast cancer (Tavassoli and Devilee, 2003). The phenotype of the untreated control sections displayed epithelial clusters of solid cells round in shape, with moderate increase in the nucleo-cytoplasmic ratio, moderate stromal desmoplasia, moderate foci of necrosis and some poorly differentiated structures. Predominantly the histopathological features observed in the untreated tumours were consistent with grade 3 while the tumours treated with variable doses of TAM were consistent with grade 2 based on modified Scarrf-Bloom-Richardson (SBR) scoring of invasive adenocarcinoma.

\section{Impact of starting tumor size on the treatment response}

In order to determine the impact of tumour size on treatment response, animals treated with TAM 100 $\mu \mathrm{g} /$ day at the starting tumour size of $10 \mathrm{~mm}$ were compared with those that received the same dose at a larger starting tumour size of $15 \mathrm{~mm}$. The final number of tumour nodules were significantly reduced in the group of animals that were initiated on treatment at $10 \mathrm{~mm}$ tumour size compared to the group of $15 \mathrm{~mm}$ starting tumour size (Table 2). A marked reduction in the end tumour volume was also observed in the $10 \mathrm{~mm}$ group, although it was not statistically significant due to the large IQR values.

No significant differences were observed when serum liver function and renal function profiles of animals treated with TAM at $10 \mathrm{~mm}$ and $15 \mathrm{~mm}$ size were compared, except for the incidental findings of high serum alkaline phosphatase level and significantly higher urea level (data not shown) in the $15 \mathrm{~mm}$ tumour group which may reflect initiation of liver and renal injuries (Usunomena et al.,2012) and therefore high tendencies of unfavorable outcome with late initiation of treatment. Likewise comparison of histomorphology of the TAM-treated 10 and $15 \mathrm{~mm}$ tumor sections was found to be essentially similar (grade 2) except for occasional comedo necrosis and reactive lymph nodes that were more prominent in the $15 \mathrm{~mm}$ compared to $10 \mathrm{~mm}$ tumours.

\section{Discussion}

Chemical-induced rat mammary tumours are commonly employed in the study of human breast cancer. The biological behavior of chemical-induced rat mammary tumours has been extensively reported (Al-Daheri et al., 2008). NMU-induced animal model is frequently used in view of its close similarities with human breast cancer in terms of ductal epithelial cells origin, malignant features, hormonal contents and significant response to ovariectomy or anti-estrogen therapy (Cardiff, 2001). Previous in vivo reports using this animal model have shown dose-dependent antitumour effect of TAM as a chemopreventive agent when administered over a long period of time (Jordan, 1983; Manni et al., 2010).

In the current study, NMU-induced mammary tumours were first developed in the animals and the tumour growth response to TAM treatment was investigated. Our results indicate that reduction in the number of tumour nodules and the end tumour volume in response to TAM treatment is dose-dependent with significant effects observed with a minimum TAM dose of $\geq 100 \mu \mathrm{g} /$ day. This is in line with a previous report on the antitumour activity of TAM $100 \mu \mathrm{g} / \mathrm{kg}$ in a similar animal model following eight weeks of treatment at the starting tumour size of $>10 \mathrm{~mm}$ (non-specified) (Goss et al., 2007). In a study analyzing the chemopreventive effect of variable doses of TAM, Gottardis and Jordan (1987) described TAM $100 \mu \mathrm{g} /$ day as an effective anti-tumour dose. In that study, partial response was demonstrated with TAM $25 \mu \mathrm{g} /$ day following eight weeks treatment and complete tumourfree state was achieved with TAM $100 \mu \mathrm{g} /$ day after 23 weeks of continous treatment. On the other hand, our study showed no significant changes in the tumour growth parameters following treatment with TAM $50 \mu \mathrm{g} / \mathrm{day}$ 
compared to the untreated group for the same treatment period. However this is in contrast to a similar report on the chemoprevention study where TAM $50 \mu \mathrm{g} /$ day was reported to maintain the animals in a complete tumourfree state after 170 days of continuous treatment (Jordan, 1983). This probably implies that while a low TAM dose can be effective as a chemopreventive agent, a higher TAM dose is required to cause regression of established mammary tumours. Alternatively, a longer treatment duration with lower TAM doses such as $50 \mu \mathrm{g} /$ day could be as effective in causing tumour regression and this represents a limitation of the current study. The phenotypic tumour changes (cribriform carcinoma) observed in the treated groups in our study is also in line with a report on the mechanism of apoptotic cell death induced in a similar animal model following 5 days treatment with Rapamycin, platelet factor 4 and their combination (Idris et al., 2014)

There was also non-uniform disparity between animals treated with variable doses of TAM and untreated group throughout the experiment. In contrast, TAM was reported to have suppressive effect on the body weight due to its selective oestrogen-like property on energy balance, thereby reducing food consumption, body fat contents and skeletal system (Fudge et al., 2009). In addition, Rodrigues and colleagues (2012) reported significant decrease in body and uterine weights of adult female rats after 30 days of daily treatment with TAM $250 \mu \mathrm{g} / \mathrm{day}$. Therefore lack of significant body weight loss in our data may be attributed to low dose of TAM administered over a short treatment period.

Previous studies involving chemoprevention or combination treatment designs have reported variable starting tumor sizes such as $5 \mathrm{~mm}$ (Ahlem et al., 2011), $6 \mathrm{~mm}$ (Melancon et al., 2005), greater than $10 \mathrm{~mm}$ (not specified) (Goss et al., 2007) and $15 \mathrm{~mm}$ (Nishino et al., 2009), at initiation of treatment with TAM or other antiestrogens. The two parallel studies of Haagg and Gould (1994) and Haagg et al., (1992) have described palpable tumour nodules of 4-5 mm and those of $\geq 10 \mathrm{~mm}$ as early and advanced mammary tumours, respectively. In addition, Jaafar and colleagues (2009) investigated the relationship between tumour size and phenotype of NMUinduced rat mammary tumours and reported that tumours of $\leq 8.0 \pm 0.5 \mathrm{~mm}$ were predominantly benign lesions, while those of tumour size $\geq 12.0 \pm 0.5 \mathrm{~mm}$ were malignant. Thus the choice of $10 \mathrm{~mm}$ and $15 \mathrm{~mm}$ starting tumor sizes in the current study is ascribed to the consideration of advanced tumor stages at these tumor sizes comparable to that of advanced human breast cancer (Cardiff, 2001).

Overall, our results indicate favorable prognosis when NMU-induced rats were initiated on treatment at the starting tumour size of $10 \mathrm{~mm}$ compared to 15 $\mathrm{mm}$. The mean number of tumour nodules at the end of TAM treatment duration was significantly less in the $10 \mathrm{~mm}$ tumour group. This is also reflected in the mean tumour volume although the difference was not found to be statistically significant due to the large intra group variability. In addition, elevation of total serum protein, urea and alkaline phosphatase in the animals initiated on treatment at $15 \mathrm{~mm}$ compared to those initiated at $10 \mathrm{~mm}$ (although statistically non-significant), may reflect a mild non-specific liver function impairment (Usunomena et al., 2012) that was however suppressed due to short treatment duration or other variable host factors.

Considering the known positive response of hormonedependent mammary tumors to antiestrogens and the pilot study nature of this report, some of the limitations therefore include lack of assessing the hormonal status of non-responding tumours and perhaps the short treatment duration. It is possible that the non-responding mammary tumours were estrogen-receptor negative (ER-ve) irrespective of starting tumour size, or perhaps better response could have been obtained from ER+ve tumours if the treatment was prolonged. On the other hand, the effect of TAM $\geq 100 \mu \mathrm{g}$ could only be suppressing the tumour growth (tumouristatic) rather than effectively killing the tumour cells (cytotoxic), and the latter may be reactivated by normal hormonal response afterwards. In view of these limitations, our observations need further validation and must be interpreted with caution.

Nevertheless, responses to the combination of a drug and a potential anticancer agent may vary between different doses of the drug used and high concentrations of the drug may also mask the activity of the potential anticancer agent. The size of the tumour at the initiation of treatment could influence the treatment outcome and variable sizes of the starting tumour have been adopted in various studies, making comparisons difficult. The current study has therefore highlighted the importance of standardizing the drug dose and the tumour size for analysis of potential anticancer agents especially when determining possible synergistic effects of multiple combinations.

\section{Acknowledgements}

We acknowledge the Universiti Sains Malaysia for providing the grant to support this study under the Research University Grant Scheme (1001.PPSP.853002). We thank Prof. Syed Hatim Noor for his advice on statistical analysis and Dr. Ch'ng Ewe Seng for his assistance on the use of imaging microscopy. We also acknowledge the Pathology department and animal house staff for their support and cooperation throughout this study. We declare that we do not have conflict of interests regarding publication of this manuscript.

\section{References}

Ahlem CN, Frincke JM, White SK, et al (2011). 17 $\alpha$-Ethynyl$5 \alpha$-androstane- $3 \alpha, 17 \beta$-diol treatment of MNU-Induced mammary cancer in Rats. Int J Breast Cancer, 2011, 61875

Al-Dhaheri WS, Hassouna I, Al-Salam S, Karam SM (2008). Characterization of breast cancer progression in the rat. Ann NY Acad Sci, 1138, 121-31.

Behtash N, Hashemi R, Karimi ZM (2009). Uterine malignancy following tamoxifen use in breast cancer patients in Iran: case series and literature review. Asian Pac J Cancer Prev, 10, 163-6.

Cardiff RD (2001). Validity of mouse mammary tumour models for human breast cancer: comparative pathology. Microsc Res Techniq, 52, 224-30.

Chan MM, Lu X, Merchant FM, Iglehart JD, Miron PL (2005). 
Hassan Muhammad Yankuzo et al

Gene expression profiling of NMU-induced rat mammary tumors: cross species comparison with human breast cancer. $J$ Carcinog, 26, 1343-53.

Chin SN, Green C, Strachan GG, Wharfe G (2014). Clinicopathologic characteristics of breast cancer in Jamaica. Asian Pac J cancer prev, 15, 3319.

Dahlui M, Ramli S, Bulgiba AM (2011). Breast cancer prevention and control programs in Malaysia. Asian Pac J Cancer Prev, 12, 1631-34.

DeSantis C, Ma J, Bryan L, Jemal A (2014). Breast cancer statistics, 2013. CA Cancer J Clin, 64, 52-62.

Fudge MA, Kavaliers M, Baird JP, Ossenkopp KP (2009). Tamoxifen and raloxifene produce conditioned taste avoidance in female rats: a microstructural analysis of licking patterns. Life Sci, 84, 282-9.

Goss PE, Strasser-Weippl K, Qi S, Hu H (2007). Effects of liarozole fumarate (R85246) in combination with tamoxifen on N-methyl-N-nitrosourea (MNU)-induced mammary carcinoma and uterus in the rat model. BMC Cancer, 7, 26.

Gottardis MM, Jordan VC (1987). Antitumor actions of keoxifene and tamoxifen in the N-nitrosomethylureainduced rat mammary carcinoma model. Cancer Res, 47, 4020-4.

Haag JD, Gould MN (1994). Mammary carcinoma regression induced by perillyl alcohol, a hydroxylated analog of limonene. Cancer Chemother Pharm, 34, 477-83.

Haag JD, Lindstrom MJ, Gould MN (1992). Limonene-induced regression of mammary carcinomas. Cancer Res, 52, 4021- 6.

Idris FM, Mansor W N A W, Irfan M, Jalal A, Jaafar H (2014). Rapamycin and PF4 induce apoptosis by upregulating bax and down-regulating survivin in MNU-induced breast cancer. Asian Pac J Cancer Prev, 15, 3939-44.

Jaafar H, Fauziah MI, Noorashikin MN (2009). The association between phenotype and size of breast tumors induced by 1-methyl-1-nitrosourea (MNU) injection in rats. Med Sci Monit, 15, 129-34.

Jordan VC (2012). A current view of tamoxifen for the treatment and prevention of breast cancer. Br J Pharmacol, 110, 507-17.

Jordan VC (1983). Laboratory studies to develop general principles for the adjuvant treatment of breast cancer with antiestrogens: problems and potential for future clinical applications. Breast Cancer Res Treat, 3, 73-86.

Karimi ZM, Behtash N, Sekhavat L, Dehghan A (2009). Effects of tamoxifen on the cervix and uterus in women with breast cancer: experience with Iranian patients and a literature review. Asian Pac J Cancer Prev, 10, 595-8.

Manni A, Xu H, Washington S, et al (2010). The impact of fish oil on the chemopreventive efficacy of tamoxifen against development of n-methyl-n-nitrosourea-induced rat mammary carcinogenesis. Cancer Prev Res, 3, 322-30.

Melancon K, Cheng Q, Kiefer TL, et al (2005). Regression of NMU-induced mammary tumors with the combination of melatonin and $9-<\mathrm{i}>$ cis $</ \mathrm{i}>$-retinoic acid. Cancer letters, 227, 39-48.

Meyer JS, Alvarez C, Milikowski C, et al (2005). Breast carcinoma malignancy grading by Bloom-Richardson system $v s$ proliferation index: reproducibility of grade and advantages of proliferation index. Mod Pathol, 18, 1067-78.

Moore MA (2013). Overview of cancer registration research in the Asian Pacific from 2008-2013. Asian Pac J Cancer Prev, 14, 4461-84.

Motamedi S, Majidzadeh K, Mazaheri M, et al (2012). Tamoxifen resistance and CYP2D6 copy numbers in breast cancer patients. Asian Pac J Cancer Prev, 13, 6101-4.

Nishino T, Ishibashi K, Hirtreiter C, Nishino Y (2009).
Potentiation of the antitumor effect of Tamoxifen by combination with the antiprogestin onapristone. J Steroid Biochem Mol Biol, 116, 187-90.

Orang E, Marzony ET, Afsharfard A (2013). Predictive role of tumor size in breast cancer with axillary lymph node involvement-can size of primary tumor be used to omit an unnecessary axillary lymph node dissection? Asian Pac J Cancer Prev, 14, 717-22.

Porter PL (2009). Global trends in breast cancer incidence and mortality. Salud Pública de México, 51, 141-6.

Rampal, Yahaya (Eds.) (2008). Cancer incidence in peninsular Malaysia, 2003-2005: the third report of the National Cancer Registry, Malaysia. National Cancer Registry.

Rodrigues-Junior DM, Lopes-Costa PV, dos Santos AR, da Silva BB, (2011). Effects of tamoxifen and raloxifene on body and uterine weights of rats in persistent estrus. Clin Exp Obstet Gynecol, 39, 362-4.

Soares-Maia R, Faustino-Rocha AI, Teixeira-Guedes CI, et al (2013). MNU-Induced Rat Mammary Carcinomas: Immunohistology and Estrogen Receptor Expression. $J$ Environ Pathol Toxicol Oncol, 32.

Tavassoli FA and Devilee P(Eds.) (2003). Pathology and genetics of tumours of the breast and female genital organs (Vol. 4). IARC Press, Lyon Cedex 08, France.

Tsubura A, Lai YC, Miki H, et al (2011). Animal models of $\mathrm{N}$-methyl-N-nitrosourea-induced mammary cancer and retinal degeneration with special emphasis on therapeutic trials. In Vivo, 25, 11-22.

Usunomena U, Ademuyiwa AJ, Tinuade OO, et al (2012). $\mathrm{N}$-nitrosodimethylamine (NDMA), Liver function enzymes, renal function parameters and oxidative stress parameters: A Review. Br. J. Pharmacol. Toxicol, 3, 165-76.

Yip CH, Taib NA, Mohamed I (2006). Epidemiology of breast cancer in Malaysia. Asian Pac J Cancer Prev, 7, 369-74 